\title{
Model of Evolution of Surface Grain Structure under Ion Bombardment
}

\author{
Anna G. Knyazeva ${ }^{1,2,3, \text { a) }}$ and Olga N. Kryukova ${ }^{1, b)}$ \\ ${ }^{I}$ Institute of Strength Physics and Materials Science SB RAS, Tomsk, 634055, Russia \\ ${ }^{2}$ National Research Tomsk Polytechnic University, Tomsk, 634050, Russia \\ ${ }^{3}$ National Research Tomsk State University, Tomsk, 634050, Russia \\ a) anna-knyazeva@mail.ru \\ b) Corresponding author: okruk@ispms.tsc.ru
}

\begin{abstract}
Diffusion and chemical reactions in multicomponent systems play an important role in numerous technology applications. For example, surface treatment of materials and coatings by particle beam leads to chemical composition and grain structure change. To investigate the thermal-diffusion and chemical processes affecting the evolution of surface structure, the mathematical modeling is efficient addition to experiment. In this paper two-dimensional model is discussed to describe the evolution of titanium nitride coating on the iron substrate under implantation of boron and carbon. The equation for diffusion fluxes and reaction rate are obtained using Gibbs energy expansion into series with respect to concentration and their gradients.
\end{abstract}

Keywords: surface treatment, ion implantation, mathematical modeling, chemical reactions, grain structure evolution, chemical potential expansion

\section{INTRODUCTION}

To provide the increase of material wear-resistance the coatings on the base of titanium nitride are widely applied. The bombardment of surface by high energy ion beams is one of the effective ways to directed structure change of composition and structure of the coating after deposition. In this case, high concentration gradient appears in coating surface that leads to essential diffusion redistribution of elements along thickness of the coating.

Experimental investigations of coating properties after treatment do not give overall notion on the processes resulting in that or other structure. In this case, a help could be awaited on mathematical modeling.

Mathematical model of modification of coating surface layer by combined particle beam was suggested in [1] taking into account new phases formation. It was demonstrated that such models are able to predict the coating composition change varying some technology parameters. The admixture in surface layer of a substrate and more detailed reaction scheme were added to the model in [2]. Structure formation had not been discussed there.

In this paper, two-dimensional mathematical model of coating treatment is formulated to describe grain structure evolution.

\section{FUNDAMENTALS}

Models of multi component media can be constructed using free energy (or other thermodynamical potential corresponding to observation condition) of heterogeneous thermodynamical system in the form (at constant pressure or stresses)

$$
G\left(C_{1}, C_{2}, \ldots, C_{n} ; T\right)=\int_{\Omega}\left(\Psi^{\text {con }}+\Psi^{\text {int }}\right) \mathrm{d} \Omega
$$


where $\Omega$ is the volume of the system under study, $C_{k}$ are mass concentrations of species:

$$
C_{k}=\frac{\rho_{k}}{\rho}, \rho=\sum_{k=1}^{n} \rho_{k}
$$

$T$ is the temperature. Gibbs energy of the system $G$ includes two parts: configurational $\Psi^{\text {con }}$ and interfacial $\Psi^{\text {int }}$. For multicomponent mixture energy density is given by

$$
\Psi^{\text {con }}=\sum_{i=1}^{n} g_{i}^{0}(T, p) C_{i}+\theta(T) \sum_{i=1}^{n} C_{i} \ln C_{i}+\frac{1}{2} \sum_{\substack{1 \leq i, j \leq n \\ i \neq j}} C_{i} C_{j} \chi_{i j}^{(0)},
$$

where the first term presents Gibbs energy of pure components; the second and the third terms correspond to mixing energy. Last sum makes possible to describe non-ideal solutions with non-lineal behavior. The part $\Psi^{\text {int }}$ is associated by many authors with nonlocal effects near the interfaces between phases in multiphase systems or between crystal grains. Contribution of nonlocal processes is connected formally with concentration gradients

$$
\Psi^{\mathrm{int}}=\sum_{i, j=1}^{n}\left(\frac{\kappa_{i j}}{2} \nabla C_{i} \nabla C_{j}\right)
$$

where parameters $\kappa_{i j}$ depend on surface energy density and thicknesses of interface layers.

If any thermodynamic potential is known as a function of thermodynamical variables, we can find all thermodynamical properties, using usual thermodynamical methods.

For example, chemical potentials of species follow the formula

$$
g_{k}=\frac{\partial}{\partial C_{k}}\left[G\left(C_{1}, C_{2}, \ldots, C_{n} ; T\right)\right]_{p, C_{j}} .
$$

Chemical potentials determine the transfer processes and chemical reaction rates.

Similar approach allows describing the evolution of grain structure in numerous applications of Phase-field method [3] and extended thermodynamics [4]. However many problems appear when transfer processes and chemical reactions define structure and properties change in real situation.

\section{PARTICULAR MODEL}

A titanium nitride coating, preliminarily deposited on substrate, is made of iron. The interface and coating external surface are plane; the particles of aluminum and boron (ions, which lose charge at surface) penetrate in coating as assumed in $[1,2]$. However, mobility of species depends on total Gibbs energy (Eq. (1)) and chemical potentials are calculated with Eq. (5). It is assumed that only one summary reaction takes place.

As a rule, 6 substances are presented in the system: Al, B, Ti, N, Fe and solid solution TiAlBN. Their concentrations are denoted as $C_{1}, \ldots, C_{6}$. There are three equation types for species. Firstly, it is the diffusion equations for particles implanted into coating

$$
\rho \frac{\partial C_{k}}{\partial t}=-\nabla \cdot \mathbf{J}_{k}+\omega_{k}+f_{k}, \quad k=1,2
$$

where $\mathbf{J}_{k}$ are diffusion fluxes; $\omega_{k}$ are the sinks of elements in chemical reaction; functions $f_{k}$ describe the sources for species due to implantation.

Secondly, the diffusion equations describe evolution of titanium and nitrogen

$$
\rho \frac{\partial C_{k}}{\partial t}=-\nabla \cdot \mathbf{J}_{k}+\omega_{k}, \quad k=3,4,
$$

Unique kinetic equation gives solution concentration 


$$
\rho \frac{\mathrm{d} C_{6}}{\mathrm{~d} t}=\omega_{6}
$$

Iron concentration follows Eq. (2).

Thermal conductivity equation due to small temperature gradients turns to heat balance equation similar to [1, 2]. Chemical reaction rate depends on concentration correspondingly to mass action law and on the temperature according to Arrhenius law. Diffusion fluxes are calculated using irreversible thermodynamics approach [5]. The detailed way of coefficients estimation is described in [6] for systems close to ideal. For Gibbs energy with additional terms (described by Eq. (3) and Eq. (4)) the approach [7, 8] is more suitable.

Boundary and initial conditions are as for $[1,2]$.

\section{DISCUSSION}

Equation for diffusion flux can be found from functional (1)

$$
\mathbf{J}_{k}=-M_{k} \nabla g_{k}=-M_{k} \nabla \frac{\delta G}{\delta C_{k}},
$$

where coefficients $M_{k}$ form mobility matrix.

Variational derivative of functional $G(y)=\int_{\Omega} L(y, \nabla y) \mathrm{d} \Omega$, where $y$ is spatially varying field, is

$$
\frac{\delta G}{\delta y}=\frac{\partial L}{\partial y}-\nabla \cdot \frac{\partial L}{\partial \nabla y} .
$$

Hence, we find

$$
\frac{\delta G}{\delta C_{i}}=g_{i}^{0}+\theta(T)\left[1+\ln C_{i}\right]+\frac{1}{2} \sum_{j \neq i} \chi_{i j}^{0} C_{j}+\frac{1}{2} \sum_{j} \nabla \cdot\left(\kappa_{i j} \nabla C_{j}\right) .
$$

Because thermal conductivity coefficient is high, temperature gradients are negligible small and phenomenon of mass transfer due to temperature gradient action is not taken into account. All coefficients can be considered as depended on composition and temperature.

As a result we obtain nonlinear differential equations for diffusing species of fourth order. Cross diffusion fluxes are also contained in these equations. Even in the simples case, when $\chi_{i j}^{0}=0, \kappa_{i j}=\kappa \delta_{i j}$ and

$$
\mathbf{J}_{k}=-M_{k}\left[\theta(T) \nabla C_{k}+\frac{1}{2} \nabla \nabla \cdot \kappa \nabla C_{k}\right],
$$

the concrete problem formulation and then its numerical realization is complicated.

For two dimensional models, differential operators take the form

$$
\nabla \ldots=\frac{\partial \ldots}{\partial x} \mathbf{i}+\frac{\partial \ldots}{\partial y} \mathbf{j}, \quad \nabla \cdot \ldots=\frac{\partial \ldots}{\partial x}+\frac{\partial \ldots}{\partial y}
$$

in Cartesian coordinate system, where $\mathbf{i}, \mathbf{j}$ are unit vectors.

Boundary conditions are not trivial. Fourth boundary conditions are necessary for fourth order equation.

If concentration is given, we have boundary condition of first type, $C_{k}=C_{k 0}$.

When flux is given, we have boundary condition of second type $\mathbf{J}_{k}=B(t)$.

Usual symmetry condition $\nabla C_{k}=0$ is required the reexamination.

The question on physical sense of more complex boundary conditions is left open.

Additional features appear when chemical reaction rate is assumed depending on chemical affinity, which is a function of chemical potentials of species participating in a reaction.

Cauchy problems are mainly discussed in literature. Computational results of some authors are quite impressive in outward appearance. However they are vulnerable to qualitative and quantitative verification, because the 
physical sense of incorporating parameters is not clear, their experimental determination is not possible today. Numerical algorithms described in literature $[9,10]$ reduce often instable, however authors of numerous papers confine them. Therefore, analysis of existing problems of missing parameters determination and development of appropriate numerical algorithms belong to our task in respect to applied problems.

\section{SUMMARY}

Thus, Gibbs energy depending on concentration of species and gradients is presented. Particular model of structure evolution of a coating under ion bombardment is described. It was shown that even the simplest case leads to equations of fourth order that demands special discussion of boundary conditions. Additionally it is necessary to find a suitable approach to calculate coefficients using independent considerations.

The work was performed in the frame of the Program of Fundamental Research of State Academies of Sciences for the period 2013-2020 Project III.23.2.1.

\section{REFERENCES}

1. N. V. Bukrina, A. G. Knyazeva, and J. Surf. Investig. X-Ray, Synchrotron Neutron Techniq. 3(1), 65 (2009).

2. O. N. Kryukova, A. G. Knyazeva, V. P. Sergeev, and A. G. Lunev, Russ. Phys. J. 55(5-2), 57 (2012).

3. M. J. Welland and D. Wolf, Phys. Rev. E 89, 012409 (2014).

4. D. Jou, J. Casas-Vazquez, and G. Lebon, Extended Irreversible Thermodynamics (Springer, 2010).

5. S. de Groot and P. Mazur, Non-Equilibrium Thermodynamics (North-Holland, Amsterdam, 1962).

6. A. G. Knyazeva, Fiz. Mezomekh. 14(6), 35 (2011).

7. D. Anders and K. Weinberg, Tech. Mech. 32, 105 (2012).

8. S. Emmanuel, A. Cortis, and B. Berkovitz, Chem. Phys. 302, 21 (2004).

9. R. R. Mohanty, "Phase-Field simulation of microstructural development induced by interdiffusion fluxes under multiple gradients", Ph.D. thesis, Orlando, USA, 2009.

10. D. Cogswell, "A phase-field study of ternary multiphase microstructures", Ph.D. thesis, Massachusetts Institute of Technology, Cambridge, USA, 2010. 